\title{
Minnesota e-Health Data Repositories: Assessing the Status, Readiness \& Opportunities to Support Population Health
}

\author{
Bree Allen $^{\star 1,2}$, Karen Soderberg ${ }^{1}$ and Martin LaVenture ${ }^{1}$ \\ ${ }^{1}$ Office of Health Information Technology, Minnesota Department of Health, Saint Paul, MN, USA; ${ }^{2}$ Council of State and Territorial \\ Epidemiologists, Atlanta, GA, USA
}

\section{Objective}

This project describes the informatics characteristics of clinical data repositories among Minnesota health systems and their opportunities and readiness to support public health practice. The focus of the study is the use of these data for public health prevention programs and surveillance, including the opportunities to address health disparities. We examine technical, organization, and process readiness of repositories in support of epidemiology and other key public health programs, and how these data can be used as a statewide public health resource.

\section{Introduction}

Health care reform and the use of electronic health record systems is dramatically changing the health care landscape creating both challenges and opportunities for public health. High adoption of health information technology among Minnesota's health care providers has created an opportunity to advance e-health by collecting and using these data to improve population health. It has been demonstrated that interoperable clinical data repositories can serve surveillance needs to support both public health and clinical care. ${ }^{1}$ Additionally, health reform is fostering the need for the collection of data to manage population health, compare and share data locally and across states for care coordination, and monitor cohorts and attributed populations. This project will provide a critical understanding of the status, challenges, and opportunities for leveraging the substantial investment in health care data systems to better support public health prevention programs, epidemiology, and surveillance to improve population health, address health disparities, and advance health equity.

\section{Methods}

An initial scan of e-health data repositories was conducted using the 2014 Minnesota hospital survey. The survey found 96 of Minnesota's 133 non-federal acute care hospitals (72\%) maintain a clinical data repository to support patient care management, population health, and/or research. A selection of these health systems are invited as key informants to participate in an individual or group interview to address each health system's e-health data repository, including the type and sources of data, levels of normalized and structured content, scope of data analytics capabilities, timeliness of data, capability for health information exchange, and readiness for use of HL7 and other standard messaging. Moreover, governance, policies, and functionality of the repository are assessed. We employ qualitative methods and examine interview notes in order to identify and code themes; narrative responses are reviewed to identify initial themes, and a second assessment identifies subthemes. Themes are used to determine readiness and opportunities for e-Health Data Repositories to support population health.

\section{Results}

Results highlight first the Minnesota health data repositories and provide a profile of data elements and structure, indications of data quality, and geographic distribution to support public health surveillance of chronic and infectious disease. Second, preliminary findings from key informant interviews reveal a wide range of models for developing and managing e-health data repositories. A minimal number of health systems use repositories for research, and data sharing agreements are not common across organizations. Barriers to sharing data tend to be associated with Minnesota's strict consent management laws and several systems noted challenges in harmonizing large and varied data sources in a timely manner.

\section{Conclusions}

E-Health data repositories have the opportunity to play a critical role in supporting population health and key epidemiologic surveillance activities. ${ }^{1}$ However, current Minnesota e-health data repositories are not used in public health practice beyond attributed populations. In order to take advantage of available data, more research is needed to help understand the types of available data, quantity, quality, and need for policy considerations to support statewide public health practice, as well as to develop a recommended framework for how these repositories can supplement existing registry programs at the Minnesota Department of Health.

\section{Keywords}

Clinical data repository; Health disparities; Informatics; Population health; Electronic health record

\section{Acknowledgments}

This study was supported in part by the Minnesota Department of Health and an appointment to the Health Systems Integration System Program Fellowship administered by CSTE and funded by the Centers for Disease Control and Prevention (CDC) Cooperative Agreement 3U38-OT000143$01 \mathrm{~S} 3$.

\section{References}

1. Grannis, S, Biondich, P, Mamlin, B, Wilson, G, Jones, L, \& Overhage, J. How Disease Surveillance Systems Can Serve as Practical Building Blocks for a Health Information Infrastructure: the Indiana Experience. AMIA Annu Symp Proc. 2005:286-90.

\section{*Bree Allen}

E-mail: bree.allen@state.mn.us 